\title{
Study to Correlate Histopathological Spectrum of Gastrointestinal Malignant Neoplasm with Demographic Profile of The Patients
}

\author{
Nital Vishnubhai Panchal and Nirav Sureshbhai Panchal* \\ Dept. of Pathology, GMERS Medical college, Gandhinagar. Gujarat - 382016. India.
}

\begin{abstract}
Background: To study occurrence, age and sex distribution, to evaluate and to correlate the various histopathological features of surgical specimens of gastrointestinal tract tumours received at pathology department of GMERS medical college, Gandhinagar (Gujarat, India).

Methods: A histopathological study of gastrointestinal tract tumours of 64 cases was carried out on surgical specimens at GMERS medical college, Gandhinagar from January-2018 to May-2019. Specimens were received in 10\% formalin.After proper fixation and grossing of the specimens tissue processing was carried out. Blocks were made, sections were cut and stained with Harris Haematoxylin and Eosin stain and observed microscopically.

Result: From the total of 64 cases: 42 Males and 22 Females wereaffected with peak occurrence in their sixth decade having anatomical distribution in oesophagus $07(10.93 \%)$, stomach $09(14.06 \%)$, small intestine $12(18.75 \%)$, appendix $01(01.56 \%)$, colon and rectum $34(53.12 \%)$ and anal canal 01(01.56\%). Histological types diagnosed were- epithelial 53(82.81\%), mesenchymal 06(09.30\%), lymphoma $04(06.27 \%)$ and neuroendocrine tumor $01(01.56 \%)$.

Conclusion: The occurrence of gastrointestinal tumours was highest in the colon and rectum accounting for 53.12\% of cases with most common variety being epithelial. The peak age distribution was in the sixth decade. Male to female ratio was 1.9:1. As Gastrointestinal tumours show a wide variation in the morphology, histopathological examination is must for the diagnosis and typing of these tumours.
\end{abstract}

Keywords: Epithelial, Mesenchymal, Lymphoma, Neuroendocrine Tumours, GIST.

\section{Introduction}

Gastrointestinal malignant tumours account for a significant proportion of all neoplasms. ${ }^{[1]}$ Colorectal cancer ranks third and stomach cancer ranks sixth among all types of cancers worldwide. ${ }^{[2]}$ Curiously the small intestine is an uncommon site for tumor despite its great length and vast pool of dividing cells. There is world wide variation in the distribution of these neoplasms which appear largely due to exogenous factors rather than genetic. ${ }^{[3]}$ The various histological types of tumours at different sites of gastrointestinal tract also differ in their incidence and prognosis.

Tumours arising from the mucosa of stomach and intestines predominate over mesenchymal and stromal tumours. Adenocarcinoma constitutes $70 \%$ of all malignancies arising in the gastrointestinal tract. Without exception, all tumours are incurable when metastasis exists. However effective treatment in case of lymphoma and stromal tumours is likely to result in cure. This study is undertaken to diagnose various histopathological types of gastrointestinal tumours.

\section{Materials and Methods}

A histopathological study of surgical specimens of gastrointestinal tract tumours of 64 cases was carried out at Histopathology department of GMERS Medical college, Gandhinagar from January 2018 to May 2019.

Specimens were received in $10 \%$ formalin. After proper fixation of the specimens, sections were taken from the lesion, its margins, surrounding tissue and lymph nodes if any. It was kept for 24 hours in $10 \%$ formalin for proper fixation; subsequently dehydration, clearing and embedding in paraffin wax were carried out. Blocks were made, sections of $3 \mu \mathrm{m}$ thickness were cut and stained with Harris Hematoxylin and Eosin stain and observed microscopically for cellular architecture, perineural and vascular invasions and surrounding areas.

Data acquired from examination of each specimen was tabulated using a performa in systematic sequence. The collected data were analyzed statistically and results obtained were compared to the existing studies in the literature.

\section{Result}

Involvement of any site from gastrointestinal tract by malignant neoplasm is possible. In present study, anatomical distribution was in oesophagus $07(10.93 \%)$, stomach 09(14.06\%), small intestine 12(18.75\%), colon and rectum $34(53.12 \%)$, anal canal $01(01.56 \%)$ and appendix $01(01.56 \%)$ as shown in table 1 . 
Out of 09 cases of gastric tumours, anatomical distributions were in body 03(33.34\%), antrum 04(44.45\%), pyloric canal $02(22.23 \%)$. Out of 12 cases affecting small intestine, tumours were found in duodenum in 04 cases (33.34\%), jejunum 02 cases (18.18\%) and ileum 06 cases (54.54\%). In 34 cases of colorectal tumours, affected areas were caecum 01 case $(02.94 \%)$, ascending colon 03 cases $(08.82 \%)$ and recto-sigmoid colon 30 cases $(88.23 \%)$.

After detailed histo-morphological (Gross and Microscopic) study, histological types diagnosed were Epithelial 53(82.81\%), Mesenchymal 06(09.30\%), Lymphoma 04(06.27\%) and Neuroendocrine tumor
$01(1.56 \%)$ as shown in table 2 . In differentiation majority of the cases were Adenocarcinoma 46 cases(71.87\%), mostly well differentiated Adenocarcinoma, while Squamous cell carcinoma, GIST and Non Hodgkin's Lymphoma constituted 07 cases (10.93\%), 06 cases $(09.37 \%)$ and 04 cases $(06.25 \%)$ respectively as tabulated in Table 3.Only one case of Carcinoid tumor was reported.

The age and gender wise distribution of Gastrointestinal Tumours is showed in Table 4. The peak age distribution was seen in $6^{\text {th }}$ decade of life with male preponderance having male to female ratio of 1.9:1.

Table 1: Anatomical distribution of Gastrointestinal Tumour.

\begin{tabular}{|l|l|l|}
\hline Site & Number & Percent (\%) \\
\hline Oesophagus & 07 & 10.93 \\
\hline Stomach & 09 & 14.06 \\
\hline Small intestine & 12 & 18.75 \\
\hline Appendix & 01 & 01.56 \\
\hline Colon and rectum & 34 & 53.12 \\
\hline Anal canal & 01 & 01.56 \\
\hline Total & $\mathbf{6 4}$ & $\mathbf{1 0 0}$ \\
\hline
\end{tabular}

Table 2: Histological types of Gastrointestinal Tumours.

\begin{tabular}{|l|l|l|l|l|l|l|l|}
\hline Histological Type & Oesophagus & Stomach & Small Intestine & Appendix & Colorectal & Anal canal & Total No. (\%) \\
\hline Epithelial & 10 & 04 & 08 & - & 30 & 01 & $53(82.81 \%)$ \\
\hline Mesenchymal & - & 02 & - & - & 04 & - & $06(9.30 \%)$ \\
\hline Lymphoma & - & 01 & 03 & - & - & - & $04(6.27 \%)$ \\
\hline Neuroendocrine & - & - & - & 01 & - & - & $01(1.56 \%)$ \\
\hline Total & $\mathbf{1 0}$ & $\mathbf{0 7}$ & $\mathbf{1 1}$ & $\mathbf{0 1}$ & $\mathbf{3 4}$ & $\mathbf{0 1}$ & $\mathbf{6 4}(\mathbf{1 0 0 \% )}$ \\
\hline
\end{tabular}

Table 3: Distribution and histo morphology of various GI tumour

\begin{tabular}{|c|c|c|c|c|c|c|c|}
\hline \multirow{2}{*}{ S. No } & \multirow{2}{*}{\begin{tabular}{|l|} 
Malignant tumours \\
(Histological Type)
\end{tabular}} & \multirow{2}{*}{ Locations } & \multicolumn{3}{|c|}{ Differentiation } & \multirow{2}{*}{ No. of cases } & \multirow{2}{*}{ Total cases $(\%)$} \\
\hline & & & Well & Moderate & Poor & & \\
\hline \multirow{4}{*}{1} & \multirow{4}{*}{ Adenocarcinoma } & Stomach & 03 & 02 & 01 & 06 & \multirow{4}{*}{$46(71.87)$} \\
\hline & & Small intestine & 07 & 01 & 01 & 09 & \\
\hline & & Colon and rectum & 13 & 16 & 01 & 30 & \\
\hline & & Anal canal & 01 & - & - & 01 & \\
\hline 2 & Squamous cell carcinoma & Oesophagus & 04 & 03 & & 07 & 07 (10.93) \\
\hline \multirow{2}{*}{3} & \multirow{2}{*}{$\begin{array}{l}\text { GIST(Gastrointestinal } \\
\text { Stromal Tumor) }\end{array}$} & Stomach & & & & 02 & \multirow{2}{*}{$06(09.37)$} \\
\hline & & Colon and rectum & & & & 04 & \\
\hline \multirow{2}{*}{4} & \multirow{2}{*}{ Non - Hodgkin's lymphoma } & Stomach & & & & 01 & \multirow{2}{*}{$04(06.25)$} \\
\hline & & Small intestine & & & & 03 & \\
\hline 5 & Carcinoid tumor & Appendix & & & & 01 & $01(01.56)$ \\
\hline Total & & & & & & 64 & $64(100 \%)$ \\
\hline
\end{tabular}


Table 4: Age and sex wise distribution of GI tumours.

\begin{tabular}{|l|l|l|}
\hline Age in years & Number of cases (Male) \% & Number of cases (Female) \% \\
\hline $0-10$ & $01(02.38)$ & $00(0)$ \\
\hline $11-20$ & $00(0.0)$ & $01(04.55)$ \\
\hline $21-30$ & $03(07.14)$ & $02(09.09)$ \\
\hline $31-40$ & $04(09.52)$ & $01(04.55)$ \\
\hline $41-50$ & $08(19.04)$ & $04(18.19)$ \\
\hline $51-60$ & $18(42.85)$ & $11(50.00)$ \\
\hline $61-70$ & $07(16.67)$ & $02(09.09)$ \\
\hline $71-80$ & $01(02.38)$ & $01(04.55)$ \\
\hline Total & $\mathbf{4 2 ( 6 5 . 6 4 \% )}$ & $\mathbf{2 2 ( 3 4 . 3 6 \% )}$ \\
\hline
\end{tabular}

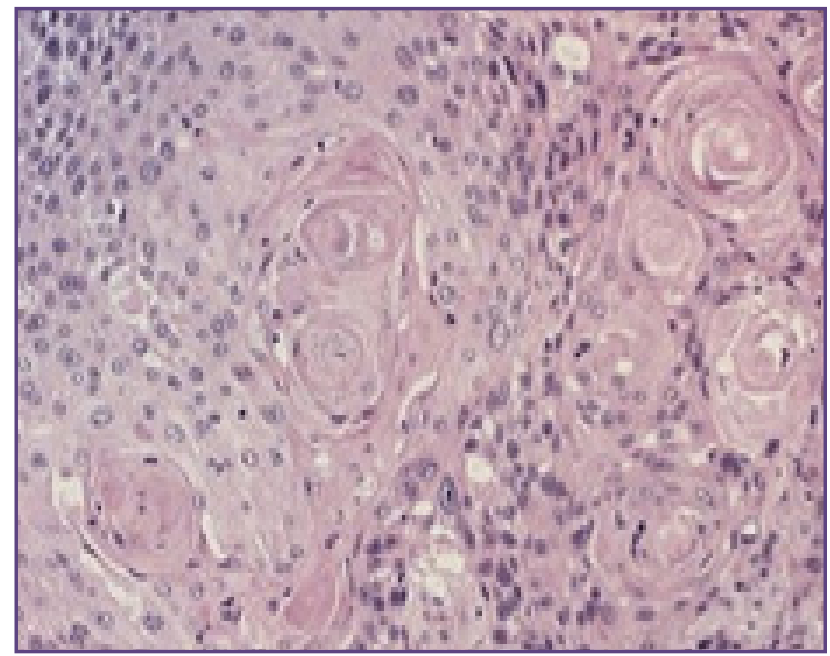

Fig. 1: Squamous cell carcinoma - Oesophagus (H \& E stain; 40X).

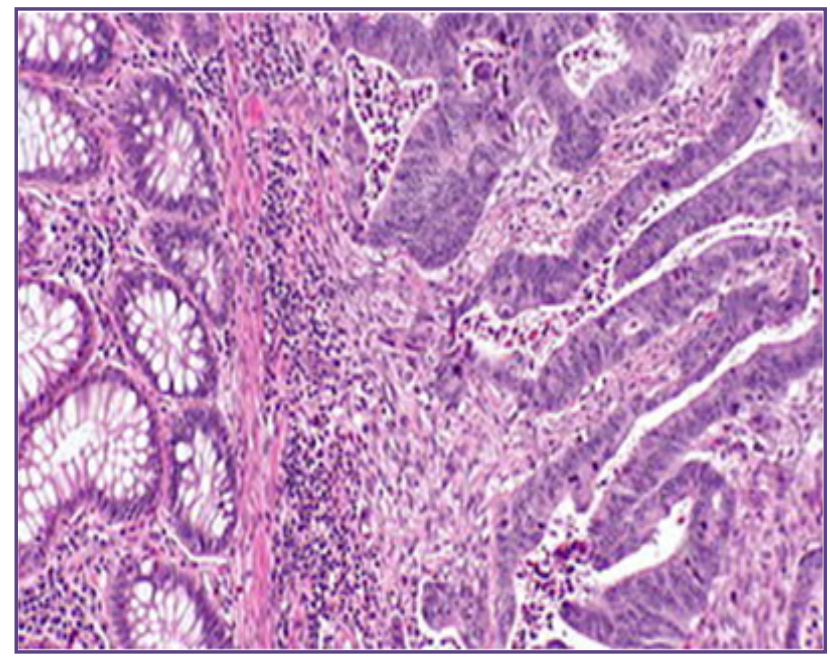

Fig. 3: Colorectal Adenocarcinoma (H \& E stain; 40X).

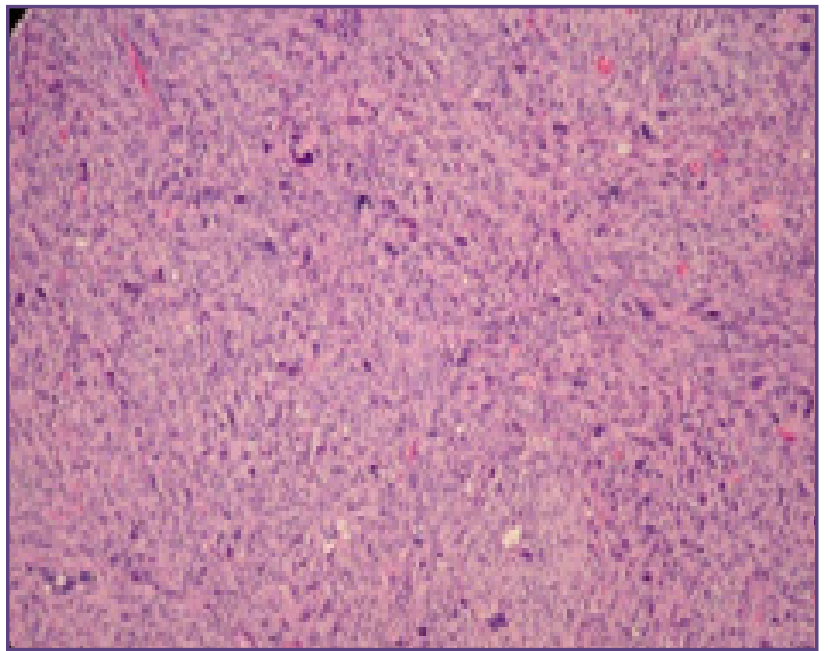

Fig. 2: GIST - Stomach (H \& E stain; 10X).

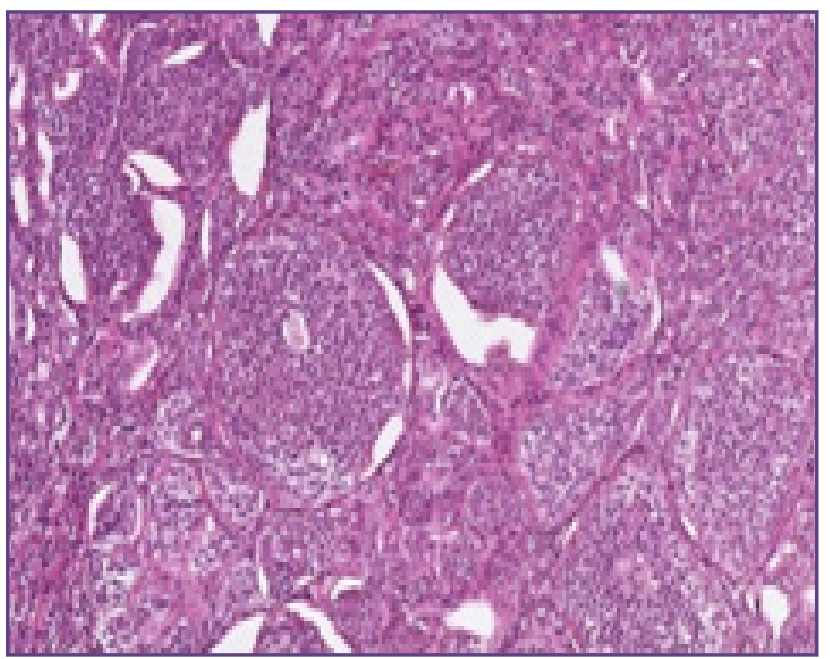

Fig. 4: Carcinoid Tumor - Appendix (H \& E stain; 40X). 


\section{Discussion}

Gastrointestinal tumours can be seen over a wide range of age (6 Months to 80 Years). In the present study, highest distribution was found in the 6th decade, which was consistent with the study by Assem O. et al,2000 $0^{[4]}$ and Patel Mandakini et al,2012 ${ }^{[5]}$ However, in the studies done by Prabhakar et $\mathrm{al}^{[6]}$ and Mohammad et $\mathrm{al}^{\left[{ }^{[7]}\right.}$, the peak age distribution was in $5^{\text {th }}$ and $7^{\text {th }}$ decade respectively. A male predominance was observed in this study with a male to female ratio of 1.9:1 which is consistent with study by Shahid Jamal et al, 2005. ${ }^{[8]}$ While Male to Female ratio is 1.67:1 and 1.39:1 in studies by Assem O. et al,2000 ${ }^{[4]}$ and Patel Mandakini et al,2012 $2^{[5]}$ respectively.

The anatomical site most commonly involved was RectoSigmoid region, constituting $53.12 \%$ of all GI tumours which is consistent with study by Leena et al. ${ }^{[9]}$, Jamal et al. ${ }^{[10]}$ and Thomas and Sobin. ${ }^{[11]}$ However in study by Abdul Kareem et al,2011 ${ }^{[12]}$ majority of cases were of transverse colon. The histological types of gastrointestinal tumours showed immense variation. However adenocarcinoma was the commonest tumor all over the gastrointestinal tract. The present study also showed similar findings.

In the oesophagus, SCC was the predominant malignant tumor. Similar finding is noted in the studies of Jamal et al. ${ }^{[10]}$ and Thomas and Sobin. ${ }^{[1]]}$ In the present study, not a single case of oesophageal adenocarcinoma was found. This could possibly be explained by the variability of predisposing factors among different population.

Among the gastric tumours, adenocarcinoma was the most predominant type. These findings were in conformity with studies conducted by Devi and Suvarna, ${ }^{[13]}$ Mohammad et $a 1,{ }^{[7]}$ and Lavanya and Sreelatha, ${ }^{[14]}$ However, higher incidence of lymphoma observed in study conducted by Lavanya and Sreelatha ${ }^{[14]}$ as compared to our study.

Adenocarcinoma was predominant tumor in small intestine which is closely correlated with Mohammad et al ${ }^{[7]}$ study. As compared to Zhou et al. ${ }^{[15]}$ we found only one case of lymphoma in small intestine.

In our study, colorectal adenocarcinoma was the commonest malignant tumor consistent with the studies of Abdul Kareem et al. ${ }^{[12]}$ and Lavanya and Sreelatha. ${ }^{[14]}$ Mucinous and signet-ring carcinoma in colorectal region comprised $10-20 \%$ in different studies ${ }^{[12,14]}$ including ours.

\section{Conclusion}

GI tumours were more common in the sixth decade. Males outnumbered females with a male to female ratio of 1.9:1. Predominantly affected site was the colorectal region. Overall, adenocarcinoma was the predominant type. Gastrointestinal tumours show a wide variation in the morphology making histopathological examination crucial for the diagnosis and typing of these tumours. As early diagnosis and treatment is beneficial for better management and is imperative in providing better quality of life to the patient, histopathological examination is must.

\section{Acknowledgements}

Authors acknowledge the immense help received from the scholars whose articles are cited and included in references of this manuscript. The authors are also grateful to authors/ editors / publishers of all those articles and journals from where the literature for this article has been reviewed and discussed. We are also grateful to all the hospital staff and technical staff without whose support it was not possible to conduct the study. No funding was received for the study. There is no any conflict of interests.

\section{References}

1. Kumar, Abbas, Fausto, Aster. Robbins and Cotran's Pathologic basis of Disease. 8th ed. Philadelphia: Saunders; 2010.P. 763-832.

2. Worldwide cancer data - Global cancer statistics for the most common cancers (2018). Retrieved from https://www.wcrf. org/dietandcancer/cancer-trends/worldwide-cancer-data.

3. Mohammad A, Makaju R. Retrospective histopathological analysis of various neoplasms of different parts of the gastrointestinal tract seen at the Kathmandu University Teaching Hospital (KUTH). Nepal, Kathamandu University Medical Journal 2005; 4: 474-478.

4. Assem O. et al. Primary gastrointestinal cancers in the Western Region of Saud Arabia. Saudi Medical Journal 2000; 21 (8): 730-734.

5. Patel MM, Gamit B, Patel PR. Analysis of gastrointestinal malignancy: A Five Years study. National Journal of Community Medicine 2012;3(3):555-557.

6. Prabhakar BR, Prabhakar H, Tung BS. Gastrointestinal malignant tumours in Amritsar (Punjab). Indian J Surg 1981; 43: 343-5.

7. Mohammad A, Makaju R. Retrospective histopathological analysis of various neoplasms of different parts of the gastrointestinal tract seen at the Kathmandu University Teaching Hospital (KUTH), Dhulikhel, Nepal. Kathmandu. Univ Med J (KUMJ) 2006; 4:474-8. 6. Jamal S, Mamoon N, Mushtaq S, Luqman M. Anal

8. Shahid J, Nadira M, Sajid M, Muhammad L, Analysis of Gastrointestinal malignancy at Armed Forces Institute of Pathology(AFIP), Rawalpindi, Pakistan. Asian pacific journal of cancer prevention 2005; 6:497-500.

9. Leena Devi KK, Suvarna N. Patterns of Gastrointestinal tumours in North Kerala. Indian Journal of Cancer 1980; 17 : 159-163. 
10. Jamal S, Mamoon N, Mushtaq S, Luqman M. Analysis of gastrointestinal malignancies at the Armed Forces Institute of Pathology (AFIP), Rawalpindi, Pakistan. Asian Pac J Cancer Prev 2005;6:497-500.

11. Thomas RM, Sobin LH. Gastrointestinal cancer. Cancer $1995 ; 75: 154-70$

12. F. B. Abdul Kareem et al. Malignant Gastrointestinal Tumours in western Nigeria: A Histopathologic analysis of 713 cases. West African Journal of Medicine 2009; 8:173-176.
13. Devi KR, Suvarna N. Pattern of gastrointestinal tumours in North Kerala. Indian J Cancer 1980;17:159-63.

14. Lavanaya M, Sreelatha R. Histopathological Study of Tumours of stomach and Intestines. Rajiv Gandhi University of Health sciences, Karnataka, Bangalore; April 2010.

15. Zhou ZW, Wan DS, Chen G, Chen YB, Pan ZZ. Primary malignant tumor of the small intestine. World $\mathrm{J}$ gastroenterol1999; 5:273-6.

*Corresponding author:

Dr. Nirav S. Panchal, 16, Amee Park society, Near Surya Ami flats, GST crossing road, Ranip, Ahmedabad. GUJARAT - 382480. INDIA

Phone: +91 9033401672

Email: niravpatho@gmail.com

Financial or other Competing Interests: None. 